\title{
Side effects of non-steroidal anti-inflammatory drugs: The experience of patients with musculoskeletal disorders
}

\author{
Onigbinde Ayodele Teslim ${ }^{1}$, M'Kumbuzi Vyvienne ${ }^{2}$, Olaogun Mathew Olatokunbo ${ }^{1}$, \\ Afolabi Joshua Oluwafisayo ${ }^{1}$, Nondwe Bongokazi Mlenzana ${ }^{3}$, Manie Shamila ${ }^{4}$, Tarimo Nesto ${ }^{5}$, \\ Mukoka Grace ${ }^{2}$ \\ ${ }^{1}$ Department of Medical Rehabilitation, College of Health Sciences, Obafemi Awolowo University, Ile-Ife, Osun State, Nigeria \\ ${ }^{2}$ Department of Physiotherapy, College of Medicine, University of Malawi, Blantyre, Malawi \\ ${ }^{3}$ Department of Physiotherapy, University of the Western Cape, Bellville, South Africa \\ ${ }^{4}$ Department of Health and Rehabilitation Science, Faculty of Health Sciences, University Cape Town, Cape Town, South Africa \\ ${ }^{5}$ Rehabilitation Technician School, Malawi Against Physical Disabilities, P.O.Box 256, Blantyre, Malawi
}

\section{Email address:}

ayotesonigbinde@yahoo.co.uk (O. A. Teslim)

\section{To cite this article:}

Onigbinde Ayodele Teslim, M'Kumbuzi Vyvienne, Olaogun Mathew Olatokunbo, Afolabi Joshua Oluwafisayo, Nondwe Bongokazi Mlenzana, Manie Shamila, Tarimo Nesto, Mukoka Grace. Side Effects of Non-Steroidal Anti-Inflammatory Drugs: The Experience of Patients with Musculoskeletal Disorders. American Journal of Health Research. Vol. 2, No. 4, 2014, pp. 106-112.

doi: 10.11648/j.ajhr.20140204.11

\begin{abstract}
Non-steroidal anti-inflammatory drugs (NSAIDs) are of choice for acute pain management in musculoskeletal disorders (MSDs) but they are not without side effects. The primary aim of this study was to investigate user's experience of side effects of oral NSAIDs among patients with MSDs. Multi-stage sampling technique was used to select 296 patients with MSDs from physiotherapy out-patient clinics of five selected hospitals. A structured and close ended questionnaire was administered to investigate the side effects experienced by respondents. Descriptive statistics (mean, standard deviation, frequency and percentages) and Chi-square were used to compare the mean percentage difference in the knowledge of NSAIDs and their side effects. Only $15 \%$ of the respondents were able to recognize the listed side effects. A significant number of the respondents $(58.7 \%)$ were not informed of the side effects of the NSAIDs at the time of prescription $\left(\mathrm{X}_{2}=\right.$ $8.82, \mathrm{p}=0.003$ ). About $33.4 \%$ and $31 \%$ of respondents identified dizziness and abdominal pain respectively as side effects. Sixty two $(22.3 \%)$ respondents had experienced dizziness while $15.9 \%$ experienced abdominal pains. Diclofenac was the most frequently prescribed NSAID (153 [51.7\%]). Only $2.0 \%$ and $2.7 \%$ of respondents were aware of phonophoresis and iontophoresis respectively as alternative means of administering NSAIDs where applicable. The Chi-square test revealed that the number of respondents that preferred alternative means of administration (where applicable) was significantly higher than those who preferred the oral routes $(\mathrm{x} 2=74.5, \mathrm{p}=0.001)$. We concluded that this sample of Nigerian patients was not informed of the side effects of NSAIDs by the prescribers. Dizziness and abdominal pains were the most experienced side effects. The role of alternative methods to administer NSAIDs need to be made available to patients where applicable to increase their health care choices.
\end{abstract}

Keywords: Side Effects, Non-Steroidal Anti-Inflammatory Drugs, Patient’s Experience, Musculoskeletal Disorders

\section{Introduction}

Musculoskeletal Disorders (MSDs) are injuries and disorders that affect the human body's movement or musculoskeletal system such as muscles, tendons, ligaments, nerves, discs and blood vessels [1]. Chronic pain as a result of MSD is a common reason for consultation in general practices [2]. Ekpenyong et al reported an incidence rate of $20.1 \%$ for MSDs among adult population of South-South Nigeria while, Adegbehingbe, et al reported a prevalence of $3.0 \%$ among 4,441 Nigerian students [3, 4].

Non-steroidal anti-inflammatory drugs (NSAIDs) are drugs of choice for acute pain management that may characterize musculoskeletal disorders including low back pain $[5,6]$. They are also commonly used in chronic musculoskeletal pain, although, the rationale remains 
controversial. There is evidence to support the use of NSAIDs in chronic spinal pain but this is based on evidence from trials lasting only few days [7 - 9]. Some researchers, however, questioned the effectiveness of NSAIDs in spinal disorders over other agents like acetaminophen, narcotic analgesics, and muscle relaxants [10 - 11]. The anti-inflammatory effect of NSAIDs are exerted through inhibition of prostaglandin $\mathrm{G} / \mathrm{H}$ synthase, or cyclooxygenase, which is the enzyme catalyzing the transformation of arachidonic acid to prostaglandins and thromboxanes [12].

Non-steroidal anti-inflammatory drugs (NSAIDs) should be avoided in patients who have established sensitivity to aspirin and should also be used with caution in hypertensive patients [13]. In a Chocrane report, the prevalence of aspirin-exacerbated respiratory tract disease was about $10 \%$ in the general population and about $21 \%$ in adults when determined by oral provocation testing [14]. In North Sydney, NSAIDs was reported to be associated with rhino-sinusitis and nasal polyps when prescribed for asthma patients [15].

Upper GI tract injury is a major side effect of NSAIDs and includes abdominal pain, gastroduodenal ulcers, mild to severe dyspeptic symptoms, haemorrhage or perforation, non-specific colitis; and other events which may lead to hospitalization or death [16]. Also, non-selective NSAID users are four to eight times more likely to develop gastroduodenal ulcers during therapy [17], [18]. There is also an increase in renal toxicity when these agents are combined with antihypertensive agents and other potentially nephrotoxic drugs [18]. Few studies have also reported hepatic reactions, hepatotoxicity and liver damage [19 - 21] Other reported risks are cardiovascular disorders, peripheral edema, hyperkalemia, particularly in patients who have diabetes, elderly patients, and patients on other hyperkalemia-inducing agents [22,23]. Patients may abstain or choose not to comply from taking oral drugs for fear of dependency and concern about these side effects.

The health care system is shifting towards shared decision making (SDM) and patients are becoming more involved in the prescription of drugs so as to enhance concordance [24 - 26]. This is an attitudinal shift in society towards greater individual autonomy and choice particularly in the United Kingdom [27, 28]. Powerful, complex and intrusive therapies demand greater understanding and active participation by the patient in decisions about therapy in order to promote greater efficacy and patient safety [29].

It is well known that the success of pharmacotherapy is highly dependent on patient's compliance and a rational choice of drug therapy regimen. A readable and correct prescription does not guarantee appropriate use by the patient [30]. Among the major principles of rational use of drugs is the need to inform the patient about pharmacological effects, side effects, potential interactions, instructions for use and warnings regarding the drug, and the diagnosis [30]. Lack of information on medication had been reported to be one of the reasons why many patients fail to take their medications as prescribed and participate more meaningfully in health care decisions that affect them [31].

Despite the side effects, physicians and orthopaedic experts still prescribe oral NSAIDs. It is not known whether the patients are aware of the hazardous effects. It is also not known if they would continue to use the medication if they experience side effects. The primary aim of this study was to investigate user's experience of side effects of oral NSAIDs among patients with MSDs. Other objectives are to investigate awareness of side effects; and to identify NSAIDs most commonly prescribed to this group of patients.

\section{Methodology}

\subsection{Study Settings}

The study settings comprised physiotherapy departments of 5 hospitals ( 3 University Teaching and 2 State). These were selected because of the presence of specialist physiotherapists in the orthopeadic unit of each physiotherapy departments in South-west, Nigeria.

\subsection{Research Design}

This study was a cross-sectional survey. The study was carried out at a particular point in time at the selected hospitals and data were collected only on the orthopaedic days at the selected settings. In a cross-sectional survey study, respondents are expected to provide a snapshot of information being inquired at that particular time [32].

\subsection{Sample}

Two hundred and ninety-six (296) patients with musculoskeletal disorder were participants in this study. The major inclusive criteria are that the patient must have at least one musculoskeletal disorder, and had been prescribed at least an oral NSAID at the time of this study. Patients who were not willing to participate in the study and or could not name the NSAID being taken were excluded from the study.

\subsection{Sample Size Determination}

According to Mathers et al, we determined a sample size of 300 patients for this study with a 5\% margin error based on assumption that the response rate would be $70 \%$ but only 296 patients met the inclusion criteria at the time of the study [32]. The hospitals were selected using sample of convenience. Multi-stage sampling technique was used to select the patients from the hospitals. The first stage involved identification of all patients with MSDs at the outpatient clinics of the selected hospitals. The second stage involved interviewing and recruiting those that were currently on NSAIDs and who could remember the name of the NSAIDs being used. 


\subsection{Instrumentation}

The instrument used in obtaining information for this study was a structured and close ended questionnaire. Prior to the study, a draft of the questionnaire was piloted among three experienced orthopaedic physiotherapists. They examined the questionnaire for simplicity, viability and precision. They also ensured that the questionnaire reflected the underlying concept and objectives of the study [33]). Changes were made based on their responses and the revised questionnaire was used for the main study. The questionnaire had four sections namely:

Section A: contained general demographic questions such as age and gender.

Section B: contained questions on their medical status (diagnosis).

Section C: assessed the awareness and side effects of oral NSAIDs experienced by respondents. The checklist of drugs in the questionnaire includes:

Piroxicam (Feldene), Diclofenac sodium (Voltaren retard), Methyl Salicylate, Menthol, Ketoprofen, Felbinac, Fluribiprofen, Ibuprofen (Ibrucap), Ketoprofen, Lidocaine, Naproxen, Ketolovac, Glucosamine sulphate (Urah) and Flotac.

Section D: sought to inquire the attitude and preference for other complementary techniques and routes of administration.

\subsection{Procedure}

Ethical approval was obtained from the ethical research committee of the Institute of Public Health, Obafemi Awolowo University, (IPH/OAU/12/196) Ile-lfe, Osun State, Nigeria. Permission was sought and obtained from the heads of the physiotherapy departments and heads of orthopaedic units at each hospital. The aims of the study were explained to respondents, and they were assured of confidentiality of information. The participants consented to participate through a written informed consent. In order to maintain anonymity, participants' names were not required in the questionnaire. The patients provided information on their medical statuses, awareness, and experiences on noticeable side effects of oral NSAIDs; knowledge and preference on other routes of administration. There was no time limit for completion of the questionnaire.

\subsection{Data Management}

The questionnaire were collated and entered into SPSS, version 17 in a private and password protected computer which only members of the research team had access to. The sample's demographic characteristics were analyzed descriptively. Chi-square was used to compare the mean percentage difference in the frequency of choice for the side effects. The cumulative percentage of respondents who could identify the side effects was computed by summation of percentages of respondents for each drug, divided by the total number of drugs. Five physiotherapists with at least 15 years experience were asked to grade proportions ranging from zero $(0 \%)$ to one hundred $(100 \%)$. The proportion refers to the sample of participants (296). The consensus proportional grading was used. The percentages were classified as:

$$
\begin{aligned}
& 0-20(\%) \text { - Very low proportion (of sample) } \\
& 21-40(\%) \text { - Low proportion } \\
& 41-60(\%) \text { - moderate proportion } \\
& 61-80(\%) \text { - Good proportion } \\
& 81-100(\%) \text { - significant proportion }
\end{aligned}
$$

\section{Results}

\subsection{Demographic Characteristics of Respondents}

Of the 296 participants, 141 were males (47.9\%) and 155 were females $(52.3 \%)$ participants who completed the survey. The mean age was $37.3 \pm 15.3$ years. Eighty six (29.1\%) respondents had postgraduate degrees, and fifty seven $(19.3 \%)$ had graduate degrees or diploma (Table 1$)$.

\subsection{Diagnoses, Prescribers and Sources of Information}

Participant's diagnoses are presented in table 2. One hundred and twenty five $(42.2 \%)$ were diagnosed at state hospitals. Two hundred and sixteen patients reported that doctors $(73 \%)$ prescribed the NSAIDs while forty one $(13.8 \%)$ nurses were reported to have prescribed the drug. The frequency distribution of other health professionals who prescribed the drugs is also presented in table 2 . One hundred and twenty-six (42.6\%) had been using the NSAIDs for more than a month; sixteen of them $(5.4 \%)$ had used it for just one week. One hundred and four (35.1\%) participants got information on side effects of NSAIDs from their doctors while $137(46.3 \%)$ read the drug pamphlets in drug pack. Other sources of information of side effects are presented in table 3.

Table 1. Qualifications and work settings of respondents

\begin{tabular}{lll}
\hline Demography & Frequency & $\mathbf{\%}$ \\
\hline Qualifications & & \\
Postgraduate & 86 & 29.1 \\
Tertiary & 57 & 19.3 \\
Secondary school & 14 & 4.7 \\
Primary school & 36 & 45.9 \\
< Primary school & 3 & 1.0 \\
Work settings & & \\
Private hospital & 67 & 22.6 \\
General hospital & 125 & 42.2 \\
Primary health centre & 30 & 10.1 \\
Self employed & 70 & 23.6 \\
Others & 4 & 1.4 \\
\hline
\end{tabular}

One hundred and eighty seven (63.2\%) respondents read the pamphlet literature in the drug pack before usage. The Chi-square test showed that the number of participants that read information contained in the pamphlets in the drug pack was significantly higher than those that did not 109 $(36.8 \%),\left(X^{2}=20.1, p=0.001\right)$. Similarly, the number of participants that understood the content of the pamphlets (188 [63.5\%]) was significantly higher than those that did 
$\operatorname{not}(73[24.7]),\left(X^{2}=203.73, \mathrm{p}=0.001\right)$.

\subsection{Awareness and Side Effects}

The frequencies of specific NSAIDs prescribed to the respondents are presented in table 4 . Ninety nine $(33.4 \%)$ respondents were able to identify dizziness as a side effect of NSAIDs while ninety-two (31.1\%) could identify abdominal pain as a side effect. The distribution for other side effects is presented in table 5. The cumulative proportion (\%) of respondents who were able to identify the listed side effects of NSAIDs was $15 \%$. In response to what side effects they had experienced, sixty two $(22.3 \%)$ had experienced dizziness while forty seven $(15.9 \%)$ experienced abdominal pains. Other experiences are also presented in table 5 .

One hundred and ninety six (66.2\%) of the respondents had experienced at least one side effect of NSAIDs (Figure 1). The result of the Chi-square test showed that the number of patients who had experienced the listed side effects was significantly higher than those who had not $\left(X^{2}=30.59\right.$, $\mathrm{p}=0.001)$.

\subsection{Preference for other Routes of Administration}

Only $2.0 \%$ and $2.7 \%$ respondents were aware of phonophoresis and iontophoresis respectively as alternative means of administering NSAIDs (Table 6).

Table 2. Diagnoses, prescribers and duration of use

\begin{tabular}{lll}
\hline Variables & Frequency & \% \\
\hline Diagnoses: Osteoarthritis & 31 & 10.5 \\
Rheumatoid arthritis & 56 & 18.9 \\
Low back pain & 56 & 18.9 \\
Fracture & 46 & 15.5 \\
Surgical intervention & 10 & 3.4 \\
Dislocation & 19 & 6.4 \\
Soft Tissue Injuries & 28 & 9.5 \\
Others & 50 & 16.9 \\
Professionals: Physicians & 216 & 73.0 \\
Pharmacists & 26 & 8.8 \\
Nurses & 41 & 13.9 \\
Others & 13 & 4.4 \\
Duration: $<1$ week & 25 & 8.4 \\
1 week & 16 & 5.4 \\
2 weeks & 49 & 16.6 \\
3 weeks & 37 & 12.5 \\
4 weeks & 43 & 14.5 \\
$>4$ weeks & 126 & 42.6 \\
\hline
\end{tabular}

One hundred and seventy nine (60.5\%) respondents reported that they are aware of other means of administering NSAIDs. The chi-square test showed that the number of participants who knew about other means of administration was significantly higher than the number of those who did not $\left(X^{2}=12.24, \mathrm{p}=0.001\right)$. One hundred and fifty-five $(52.4 \%)$ would not like to continue with the medication, this number was significantly higher than those who wished to continue $\left(X^{2}=141.7, p=0.001\right)$, (Table 7).
Table 3. Sources of information on side effects

\begin{tabular}{lll}
\hline Media & Frequency & Percentages \\
\hline Doctor & 104 & 35.1 \\
Nurse & 44 & 14.9 \\
Literature & 41 & 13.9 \\
Radio & 22 & 7.4 \\
TV & 45 & 15.2 \\
Internet & 35 & 11.8 \\
Friend & 48 & 16.2 \\
Pamphlet in drug box & 137 & 46.3 \\
\hline
\end{tabular}

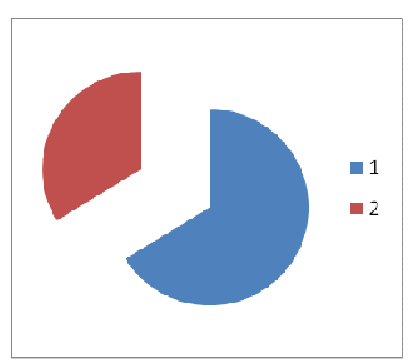

1. Experienced 2. None experienced

Figure 1. Experienced side effects

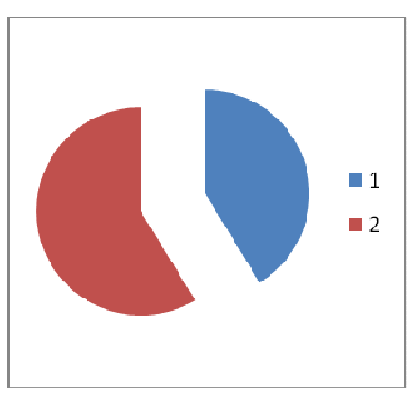

1. Informed 2. Not informed

Figure 2. Information on side effects

Two hundred and twenty three (75.3\%) would prefer alternative means of administering NSAIDs considering the benefits. The Chi-square test result revealed that the number of participants that preferred alternative means of administration (where applicable) was significantly higher than those who preferred the oral route $\left(\mathrm{x}^{2}=74.5, \mathrm{p}=0.001\right)$, (Table 7).

Table 4. Distribution of NSAIDs prescribed

\begin{tabular}{lll}
\hline NSAIDs & Frequency & \% \\
\hline Piroxicam & 100 & 33.8 \\
Diclofenac Sodium & 153 & 51.7 \\
Methyl Salicylate & 114 & 38.5 \\
Menthol & 72 & 24.3 \\
Ketoprofen & 24 & 8.1 \\
Felbinac & 31 & 10.5 \\
Fluribiproen & 15 & 5.1 \\
Ibuprofen & 120 & 40.5 \\
Ketoprofen & 6 & 2.0 \\
Lidocaine gel & 3 & 1.0 \\
Naproxen & 18 & 6.1 \\
Ketolovac & 1 & 0.3 \\
Glucosamine sulphate & 46 & 15.5 \\
Flotac & 21 & 7.1 \\
\hline
\end{tabular}




\section{Discussion}

Most respondents had been using the medication for more than three weeks; we assumed that this period would have given them the opportunity to notice side effects. A significant proportion was able to read and understand the literature pamphlets in the drugs pack. This might be attributed to the tertiary educational and post graduate qualifications of most respondents. Wahinuddin et al reported that factors such as knowledge, attitudes and motivation are important for patient compliance; and the effectiveness of therapy and that these factors might be influenced by the patient's education level, gender, the type of the disease and medication, as well as doctor-patient relationship, and culture [34]. The majority of participants (73\%) were placed on NSAIDs by medical practitioners. It is only in the UK that specially trained non-medics were licensed as supplementary prescribers but we observed that other health professionals who were none prescribers also prescribed for patients [35]. The finding of inadequate information on side effects of NSAIDs by prescribers was a similar trend observed by Wynne and Long who also reported that $67 \%$ of participants were not informed of the side effects of NSAIDs [36]. Also, Mcmahon et al reported that doctors did not provide sufficient information on adverse effects of drugs and no reason was adduced for this [37].Yilmaz et al reported that only half of the patients with osteoarthritis in Turkey were informed of the side effects of NSAIDs by their doctors [38]. However, current findings contradicted those of Cottrell et al who reported that participants in their study were informed of the side effects of NSAIDs prior to prescription [39].

Furthermore, almost half of respondents got information on side effects by reading drug literature inserted in the packs while few got it from TV $(15.2 \%)$ and radio broadcast $(7.4 \%)$. This is similar to the findings of Wahinuddin et al who also reported that $15 \%$ obtained information by surfing the internet and 9.2\% from printed media in rheumatology clinic at Northern Malaysia [34].

Table 5. Side effects recognized and experienced

\begin{tabular}{lllll}
\hline \multirow{2}{*}{ Side effects } & Awareness & \multicolumn{3}{c}{ Experienced } \\
\cline { 2 - 5 } & Frequency & \% & Frequency & \% \\
\hline High blood pressure & 56 & 18.9 & 26 & 8.8 \\
Low blood pressure & 59 & 19.9 & 12 & 4.1 \\
Indigestion & 75 & 25.3 & 38 & 12.8 \\
Gastro-intestinal bleeding & 18 & 6.1 & 2 & 0.7 \\
Ulcer & 69 & 23.3 & 30 & 10.1 \\
Vomiting & 52 & 17.6 & 32 & 10.8 \\
Diarrhea & 26 & 8.8 & 15 & 5.1 \\
Abdominal pain & 92 & 31.1 & 47 & 15.9 \\
Heart attack & 14 & 4.7 & 20 & 0.7 \\
Kidney disorders & 20 & 6.8 & 3 & 1.0 \\
Dizziness & 99 & 33.4 & 62 & 22.3 \\
Comma/unconsciousness & 14 & 4.7 & 2 & 0.7 \\
Nausea & 65 & 22.0 & 37 & 12.5 \\
Constipation & 38 & 12.8 & 22 & 7.4 \\
Gastric pain & 25 & 8.4 & 14 & 4.7 \\
Liver problems & 11 & 3.7 & 3 & 1.0 \\
Others & 6 & 5.4 & 7 & 2.4 \\
\hline
\end{tabular}

Table 6. Awareness on routes/techniques

\begin{tabular}{lll}
\hline Demography & Frequency & \% \\
\hline Transcutaneous & 53 & 17.9 \\
Injection & 73 & 58.4 \\
Transdermal massage & 115 & 38.9 \\
Lingual & 13 & 4.4 \\
Iontophoresis & 8 & 2.7 \\
Phonophoresis & 6 & 2.0 \\
\hline
\end{tabular}

Table 7. Attitude and preference for routes

\begin{tabular}{llllll}
\hline & & Freq & \% & X2 & P \\
\hline \multirow{2}{*}{ Read drug pamphlet } & YES & 187 & 63.2 & \multirow{2}{*}{20.1} & \multirow{2}{*}{0.001} \\
Understood pamphlet & NO & 109 & 36.8 & & \\
info & NES & 188 & 63.5 & 203.7 & 0.001 \\
Continuation of & YES & 120 & 40.5 & & \\
NSAID & NO & 155 & 52.4 & 141.7 & 0.001 \\
Knowledge of other & YES & 179 & 60.5 & & \\
means & NO & 117 & 39.5 & 12.2 & 0.001 \\
Preference for other & YES & 223 & 75.3 & & \\
means & NO & 73 & 24.7 & 74.5 & 0.001 \\
\hline
\end{tabular}

*Based on valid $\%$ of those who responded to the questions

Diclofenac (voltaren retard) was the most frequently prescribed NSAID followed by ibuprofen (ibrucap) and this is consistent with the findings of Albsoul-Younes et al in a Jordanian population [40]. Diclofenac has been noted to be the most widely used NSAID in the world and this is despite hepatic reactions, hepatotoxicity and liver damage reported in several studies [19 - 21]. The usage might be attributed to its efficacy in pain management. It is also the most widely studied in reference to musculoskeletal disorders [41].

Dizziness, abdominal pain, indigestion and gastric ulcers characterized the most frequently experienced and recognized side effects of NSAIDs, although, very few were aware of the listed side effects. The study by Yilmaz et al, reported that most of the participants (84.5\%) knew about dyspeptic disorders (characterized by stomach pains, indigestion, nausea) as side effects of NSAID [38]. We found that the number of patients who had experienced the listed side effects was significantly higher than those who had not. This is similar to results from a study by Cottrell et al and Albsoul-Younes et al, who reported that more than half of participants $(67 \%)$ in their studies had experienced at least one side effect $[39,40]$.

The role of alternative methods aside oral and injections need to be available to patients where applicable to increase their health care choices as most respondents would prefer to discontinue with NSAIDs if other effective options with minimal or no side effect are available. Although, most respondents were unaware of alternative means of administering NSAIDs apart from oral and injections. However, Carnes et al reported that most patients on medication would be willing to continue with the drugs despite the side effects [42].

We felt the preference of most respondents for administration through injection might be a difficult task considering the daily use of the drug as this might necessitate daily hospital visits. Contrarily, Albsoul-Younes 
et al found that $74 \%$ of participants in their study preferred the oral route while only $9 \%$ preferred injections [40]. The majority of patients $(75.3 \%)$ would prefer other alternative of administering NSAIDs using procedures of iontophoresis and phonophoresis where applicable if they would reduce adverse reactions, although, very few knew about these procedures.

Gastro-intestinal disorders are the commonest adverse effects of NSAIDs, hence, there is need to know the medical and social profile of patients and also identify risk factors. Rusell observed that patients over 70 years, underlying respiratory dysfunction, cardio-vascular disorder, history of peptic ulceration and smoking are risk factors for developing gastro-intestinal disorders following NSAIDs prescription [16, 43]. These will guide dose adjustment, choice of alternative drugs (effective but with minimal side effects), dietary advice and co-prescription of suppressants which may reduce the adverse reaction of NSAIDs. This study did not take cognizance of the total population of patients with musculoskeletal disorders at the selected hospital settings and the findings can't also be generalized; this might be a limitation of this study. Similarly, the sources or manufacturers of the drugs were not considered. This is also another limitation because drugs adulteration can also increase side effects.

\section{Conclusion}

Diclofenac was the most prescribed NSAID. A significant number of the patients with MSDs reportedly experienced side effects which were mostly dizziness, abdominal pain, indigestion and gastric ulcers from NSAIDs. Most of them were not informed and were unaware of the side effects. Only $2.7 \%$ and $2.0 \%$ of respondents were aware of iontophoresis and phonophoresis respectively as alternative means of NSAIDs administration. The cost of the availability of alternative means should be a focus for future studies.

\section{References}

[1] Middlesworth Mark (2013). Definition of musculoskeletal disorder; ergonomic plus 1.765.384.4499.

[2] Annika, V. Nikita, J. Corrina, L. Markus, B. Konstantin, S. Jurgen, B. Annette, B. (2012). Differences between patients with chronic widespread pain and local chronic local pain in primary care- a comparative cross-sectional analysis. BMC Musculoskeletal Disorders 14: 351.

[3] Ekpenyong, C. E1, Udokang, N.E1, Akpan, E.E, Samson. (2012).Double Burden, Non-Communicable Diseases And Risk Factors Evaluation In Sub-Saharan Africa: The Nigerian Experience. European Journal of Sustainable Development; 1, 2, 249-270.

[4] Adegbehingbe O.O., Fatusi A.O., Adegbenro C.A., Adeitan O.O., Abass G.O., Akintunde A.O.(2009). January Musculoskeletal disorders: Epidemiology and treatment seeking behavior of secondary school students in a Nigerian community. Indian J community med.; 34(1): 52-56.
[5] Schnitzer TJ, Ferraro A, Hunsche E. (2004). A comprehensive review of clinical trials on the efficacy and safety of drugs for the treatment of low back pain. J Pain Symptom Manage; 28:72-95.

[6] Ekman EF, Koman LA. (2005). Acute pain following musculoskeletal injuries and orthopaedic surgery:mechanisms and management. Instr Course Lect; $54: 21-33$.

[7] Hickey RF (1982). Chronic low back pain: a comparison of diflunisal with paracetamol. NZ Med J; 95:312-4.

[8] Videman T, Osterman K. (1984). Double-blind parallel study of piroxicam versus indomethacin in the treatment of low back pain. Ann Clin Res; 16:156-60.

[9] Berry H,Bloom B, Hamilton, EB. (1982). Naproxen sodium, diflunisal, and placebo in the treatment of chronic back pain. Ann Rheum Dis; 41:129-32.

[10] Peloso P, Gross A, Haines T. (2005). Medicinal and injection therapies for mechanical neck disorders. The Cervical Overview Group. Cochrane Database Syst Rev; 2: CD000319.

[11] Brunton L, Lazo J, Parker K (2006). Goodman \& Gilman's the pharmacological basis of therapeutics.11th ed. New York: McGraw-Hill Companies.

[12] Vane JR, Botting RM. (2003). The mechanism of action of aspirin. Thromb Res; 110: 255-8.

[13] Weir MR. (2002). Renal effects of non - selective NSAIDs and coxibs Cleve. Clin J Med; 69(Suppl 1):SI53-8.

[14] Gollapudi RR, Teirstein PS (2004). Aspirin sensitivity: implications for patients with coronary artery disease. JAMA; 292:3017-23.

[15] Jenkins C, Costello J, Hodge L (2004). Systematic review of prevalence of aspirin induced asthma and its implications for clinical practice. BMJ; 328:434-6.

[16] Russell R I (2001). Non-steroidal anti-inflammatory drugs and gastrointestinal damage-problems and solutions Postgrad Med J 2001;77:82-88

[17] Goldstein JL,Silverstein FE, Faich G (2000). Gastrointestinal toxicity with celecoxibvsnonsteroidal antiinflammatory drugs for osteoarthritis and rheumatoid arthritis. The class study: a randomized controlled trial. JAMA; 284: 1247-55.

[18] Rostom A, Dube C, Wells G (2002). Prevention of NSAID induced gastroduodenal ulcers. Cochrane Database Syst Rev; 4: CD002296.

[19] Brun, J.; Jones, R (2001), Nonsteroidal Anti-Inflammatory Drug-Associated Dyspepsia: the Scale of the Problem. Am. J. Med.110, 12S-13S.

[20] Kearney, P. M.; Baigent, C.; Godwin, J.; Halls, H.; Emberson, J. R.; Patrono, C (2006). Do Selective CycloOxygenase-2 Inhibitors and Traditional Non-Steroidal AntiInflammatory Drugs Increase the Risk of Atherothrombosis? Meta-Analysis of Randomised Trials. BMJ 332, 1302-1308.

[21] Vonkeman, H. E.; Brouwers, J. R.; van de Laar, M. A. (2006). Understanding the NSAID Related Risk of Vascular Events. BMJ, 332, 895-898. 
[22] KrotzF, Schiele TM, Klauss V (2005). Selective COX-2 inhibitors and risk of myocardial infarction. $J$ Vasc Res; $42: 312-24$

[23] Brater DC, Harris C, Redfern JS (2001). Renal effects of COX-2-selective inhibitors. Am J. Nephrol; 21:1-15.

[24] Emanuel, E J \& Emanuel, L L. (1992) Four models of the physician-patient relationship. Journal of the American Medical Association. 267(16), 2221-6.

[25] Charles C, Gafni A \& Whelan T. (1997) Shared decisionmaking in the medical encounter: What does it mean? (or it takes at least two to tango). Social Science and Medicine 44(5), 681-692.

[26] Charles C, Gafni A, \& Whelan T. (1999) Decision-making in the physician-patient encounter:revisiting the shared decision-making model. Social Science and Medicine 49, 651-661.

[27] Department of Health. (2001). The Expert Patient: a new approach to chronic disease management in the 21 st century. London: Department of Health.

[28] Wanless D. (2002). Securing our future health: taking a long term view. London: HM Treasury

[29] Donaldson L. (2003). Expert patients usher in a new era of opportunity for the NHS. BMJ; 326:279-80. PMC free article. PubMed

[30] De Vries T.P.G.M., Henning R.H., Hogerzeil H.V., Fresle D.A. (1993). Guide to good prescribing; WHO/DAP/94.11.

[31] Popovich NG. Ambulatory patient care. In: Gennaro AR, ed. (1985). Remington's pharmaceutical sciences, 17th ed. Pennsylvania: Mack Publishing Company, 1681-701.

[32] Mathers Nigel, Nick Fox,Amanda Hunn (2009) Surveys and Questionnaires. The NIHR Research Design Service for Yorkshire \& the Humber. www.rds-eastmidlands.nihr.ac.uk

[33] García de Yébenes Prous M. Jesús, a, * Francisco Rodríguez Salvanés, b and Loreto Carmona Ortells (2009). Validation of questionnaires. Reumatol Clin. 2009;5(4):171-177.
[34] Wahinuddin Sulaiman, Ong Ping Seung, Rosli Ismail.Patient's Knowledge and Perception Towards the use of Non-steroidal Anti-Inflammatory Drugs in Rheumatology Clinic Northern Malaysia. Oman Medical Journal (2012) Vol. 27, No. 6: 505-508

[35] Brown JB, Stewart MA, McCracken E, et al. The patientcentred clinical method, 2: definition and application. FamPract. 1986;3:75-79.

[36] Wynne HA, Long A (1996). Patient awareness of the adverse effects of non-steroidal anti-inflammatory drugs (NSAIDs) Br J Clin Pharmacol.; 42:253-256. [PMC free article] [PubMed]

[37] McMahon T, Clark C.M, Bailie G R: Who provides patients with drug information? Br Med J (Clin Res Ed); 294(6568): $355-356$

[38] Yilmaz H, Gürel S, Ozdemir O (2005). The use and safety profile of non-steroidal antiinflammatory drugs among Turkish patients with osteoarthritis.Turk J Gastroenterol. Sep;16(3):138

[39] Cottrell N, Emmerton L, DenaroC (2007) Heart failure patients' awareness and perceptions of the risk of using NSAIDs: a pilot study.ClinTher. Apr; 29 (4):717-9.

[40] Albsoul-Younes AM, Jabateh SK, Abdel-Hafiz SM, Al-Safi SA (. 2004). Awareness and frequency of potential side effects on nonsteroidal anti-inflammatory drugs among the Jordanian patient population. Saudi Med J. Jul; 25(7):907-11

[41] Nair Bindu and Taylor Regina (2010). A Review of Topical Diclofenac Use in Musculoskeletal Disease. Pharmaceuticals, 3, 1892-1908.

[42] Carnes D, Anwer Y, Underwood M, Harding G, Parsons S (2007). Influences on older people's decision making regarding choice of topical or oral NSAIDs for knee pain: qualitative study.BMJ. 2008 Jan 19; 336(7636):142-5. Epub Dec 4.

[43] Russell RI (1999). Defining patients at risk of NSAID gastropathy. Ital J Gastroenterol Hepatol; 31(suppl 1 S):14 18 